\title{
EVALUATION OF IMMEDIATELY LOADED KNIFE THREAD IMPLANTS FOR THE REPLACEMENT OF MANDIBULAR SINGLE- ROOTED TEETH (CLINICAL \& RADIOGRAPHIC STUDY)
}

\author{
Nourhan M. Abd El-Moneim ${ }^{l} B D S$, Samraa A. El Sheikh² $P h D$, Sherif A. Tehemar ${ }^{3} P h D$
}

\begin{abstract}
INTRODUCTION: Modern implantology is changing direction towards the development of new implant macrodesigns that are able to provide a high level of physico-mechanical characteristics and can offer a high level of primary implant stability that is important for longterm success of the immediate loading treatment protocols.

OBJECTIVES: Clinical and radiographic evaluation of immediately loaded knife thread implants for the replacement of mandibular singlerooted teeth.

MATERIALS AND METHODS: This study was conducted on ten adult patients with missing mandibular single rooted teeth. AnyRidge knife threaded tapered implants were inserted and immediately loaded with a non-occluding temporary crowns immediately after implant placement. After 3 months, final crowns were delivered. All implants were followed for 6 months. Clinically, each patient was evaluated for pain, tenderness or discomfort, presence of swelling or infection and mobility of the implant. Radiographically, standardized periapical x-ray films were taken then Image-J processing Program was used for the assessment of marginal bone level and bone density around the implant. RESULTS: In only one case moderate pain, peri-implant infection with suppuration and grade III mobility was registered in the first follow up month, that was regarded as a failure case and the implant was removed, resulting in implant survival rates of $90 \%$ after six months. There was a statistically significant increase in bone density around the implants throughout the evaluation period. The mean of peri-implant bone loss increased from immediately post-operative to the $3^{\text {rd }}$ month. This increase was statistically significant. Then there was statistically insignificant decrease from the $3^{\text {rd }}$ to the $6^{\text {th }}$ months.

CONCLUSIONS: Immediate non-occlusal loading of knife thread implants for the replacement of mandibular single rooted teeth was a successful treatment procedure, with satisfactory clinical outcomes, good survival rate $(90 \%)$ and a low incidence of complications.

KEY WORDS: Immediate loading, Knife threads, Dental implant, Nanostructured calcium-incorporated surface, Implant design, Thread design.
\end{abstract}

1- Dentist, Ministry of Health, Faculty of Dentistry, Alexandria University, Alexandria, Egypt.

2- Professor, Department of Oral and Maxillofacial Surgery, Faculty of Dentistry, Alexandria University, Egypt.

3- Assistant Professor, Department of Oral and Maxillofacial Surgery, Faculty of Dentistry, Alexandria University, Egypt.

\section{INTRODUCTION}

Increasingly, implant patients have been demanding treatment protocols that take less time, require fewer surgeries and deliver superior function and aesthetics. In response, the growing popularity of immediate loading protocols has occurred (1).

Today immediate loading (IL) of dental implants is an eminent and acknowledged treatment strategy which is extensively being used for the rehabilitation of missing teeth. IL has gained popularity due to less tissue trauma, reduced overall treatment time, decreased patient's anxiety and discomfort, high patient acceptance as well as better function and aesthetics (2).

Although excellent results have been reported for immediately loaded implants, these protocols are technique sensitive and the results still a challenge (3). Success depends upon many factors, including the achievement of high primary implant stability $(4,5)$.

Primary stability is a critical factor that determines the long-term success of dental implants. It is responsible for preventing the micromotion of the implant in the bone site, promoting natural healing and effective bone formation until adequate biological stability has been established. Primary stability of dental implants is highly dependent on implant design, surgical technique and the bone density and quality $(6,7)$.
In order to shorten the healing time, the strategy is to alter the biocompatibility of titanium implant surfaces, modifying the surgical technique and changing the implant design to improve initial bone-to-implant contact (BIC) $(8,9)$.

The implant body design has been modified over the years to enhance primary stability, particularly for immediate loading, as the implant requires maximum stability at the time of the placement (10).

Such modifications were; increased implant diameter and length, modification of number of threads, type of threads and taper of the body of the implant. All these modifications have the common goal to increase the implant surface area in contact with the surrounding bone and engage marginal and lateral cortical bone to a greater extent (11).

It is demonstrated that threads have been incorporated into implants to improve the initial contact between implant and bone, enlarge implant surface area and distribute stresses favorably. Implants with more threads achieved greater implant primary stability values (12).

Recently, Knife thread implant design was developed. It was hypothesized that Knife thread design results in maximum BIC, high primary stability, maximized compressive force resistance and minimized shear force production (13).

The present study therefore aims to evaluate clinical performance of a recently developed implant with a specific 
Knife thread design when immediately loaded for replacement of mandibular single rooted teeth.

\section{MATERIALS AND METHODS \\ Study design}

This study was conducted on ten adult patients having missing mandibular single rooted teeth indicated for implant placement using a one-stage surgery with immediate loading protocol. Patients were selected from the Outpatient clinic of the Oral and Maxillofacial Surgery Department, Faculty of Dentistry, Alexandria University.

\section{Inclusion Criteria}

- Patients' ages ranged between 20-40 years.

- Missing mandibular single rooted teeth.

- Good systemic and oral health.

- Sufficient residual bone and adequate interocclusal space at the edentulous area to allow implant placement and its future crown restoration.

- Patients psychologically accepted the implant and the involved procedures and able to sign an informed consent form.

\section{Exclusion Criteria}

- Parafunctional habits such as bruxism and clenching.

- Insufficient inter-occlusal space.

- Insufficient bone volume.

- Medical condition or medication that might compromise healing or osseointegration such as uncontrolled diabetes mellitus and osteoporosis.

- Poor oral hygiene.

- Habits that might reduce the blood flow and retard healing such as heavy smoking and alcoholism.

\section{Informed consent}

All patients received thorough explanations about the planned treatment and its potential risks and complications, and signed a written informed consent form prior to being enrolled in the study. It was also mentioned that the patient had the right of withdrawal from the study anytime without any consequences. Ethical approval for this study was obtained from the research ethics committee, Faculty of Dentistry, Alexandria University before beginning the study.

\section{Materials}

Implant System: AnyRidge Implant System (MegaGen Implant Co. Ltd., Gyeongbuk, South Korea). The implants used in this study are characterized by a tapered design with strong self-cutting Knife threads which have thin, sharp, and round faced flutes and rounded, non-cutting edges (Fig 1). The implants feature a nanostructured calcium-incorporated (Xpeed) titanium surface $(14,15)$. The implants for the study were available in lengths of $10 \mathrm{~mm}, 11.5 \mathrm{~mm}, 13 \mathrm{~mm}$; the available diameters were $3.5 \mathrm{~mm}, 4.0 \mathrm{~mm}$ and $4.5 \mathrm{~mm}$.

\section{SURGIC XT PLUS}

implant micromotor system (NSK, Nakanishi Inc., Tochigi, Japan) with advanced torque calibration $(50 \mathrm{Ncm})$.

\section{Pre-surgical Phase}

Prior to implant placement, each patient was investigated clinically and radiographically. All patients were subjected to a detailed history taking including: personal data, medical history and dental history. Clinical evaluation of the implant site including inspection, palpation of the edentulous alveolar ridge, the occlusion, and inter-occlusal space.

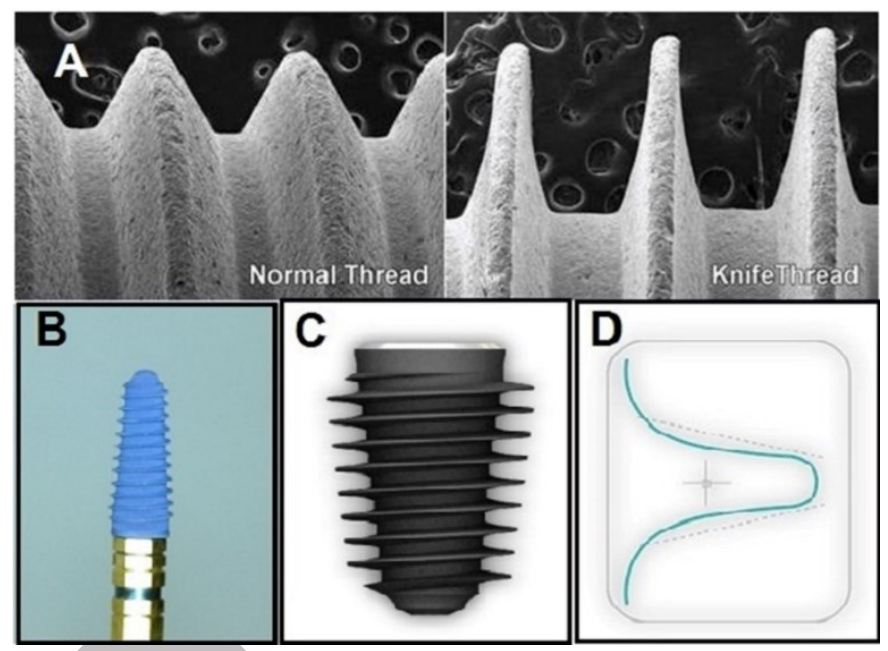

Figure 1: A. Microscopic view of a standard V-shape thread design (left) and the novel knife thread design (AnyRidge; right). B. Clinical view of AnyRidge implant. C. AnyRidge dental implants. D. knife thread design with rounded faced flutes

Primary alginate impressions for both arches were taken and diagnostic study models were prepared and duplicated. Study casts were used for the evaluation of the jaw relationship and the inter-occlusal space, construction of surgical guide stent and fabrication of provisional acrylic restorations. On the study cast, a selected acrylic tooth on the area of the missing tooth was placed, and was sealed with the carding wax. Following this, a silicone putty index was made involving at least one tooth beyond it on each side. Build up the implant abutment on the model with the carding wax was done and an impression was taken then it was poured by stone. Using a previously fabricated putty index, a provisional acrylic crown was fabricated free from occlusion.

Pre-operative orthopantomogram (OPG) was done for all patients to detect any clinically undetectable pathology and approximation to important anatomical structures and were further supplemented with standardized periapical radiographs for the implant site to more accurately assess the quantity of bone available for implant placement.

A graduated bone caliber device was used to determine the buccolingual diameter of bone at the implant site to select the suitable implant diameter. Few drops of local anesthesia were placed in the buccal and lingual sulcus opposite to the implant site then a bone caliber was used to measure the available bone width from the facial cortical plate to the lingual cortical plate at the crest of the prospective implant site.

All patients received professional oral hygiene treatment prior to implant insertion. Preoperative oral antibiotic one hour before surgery was given Amoxicillin $875 \mathrm{mg}$ /clavulanic acid 125mg (Augmentin 1gm Tablets, Medical Union Pharmaceuticals (MUP), GlaxoSmithKline (gsk), Cairo, Egypt) and $0.12 \%$ chlorohexidine gluconate mouth wash (Hexitol mouthwash, Arab drug company, Cairo, Egypt) was used to rinse for 30 seconds before operation.

Surgical Phase (Fig 2, 3)

All patients were operated under local anaesthesia. Crestal incision was performed and full thickness mucoperiosteal flap was reflected to access the site. Surgical guide stent was adapted to the teeth and the pilot drilling was performed with the lance drill ( $2 \mathrm{~mm}$ diameter) through the stent hole to confirm the exact drilling location and direction. The 
sequence of drilling was performed as recommended by the manufacturer, and parallelism was checked using direction indicator. Profuse normal saline irrigation was used throughout the drilling procedure. The implant was inserted into the osteotomy site using SURGIC XT PLUS implant micromotor system (NSK, Nakanishi Inc., Tochigi, Japan). The insertion torque was set at $35 \mathrm{Ncm}$ and was progressively increased as the implant stopped turning or leaving one or two threads above the alveolar crest then the implants were manually seated in the proper position, at crestal level, using a ratchet wrench till the implant was fully seated and flushed with bone and their stability was determined clinically as the absolute absence of axial or rotational movement by the removal of the implant driver without use of the stabilizing wrench. Implant abutment was hand-tightened onto the implant with finger pressure and fixed by the abutment screw. The flap was sutured with interrupted sutures. Final preparation of the abutment was completed with carbide bur at high speed with profuse irrigation. Immediate loading of the implant with a provisional acrylic crowns which were prepared to be $2 \mathrm{~mm}$ free from occlusion and then placed using a thin layer of temporary cement. A Standardized periapical radiograph was made immediately after implant placement to evaluate implant position and ensure proper abutment placement and as a baseline measurement for peri-implant marginal bone level measurements.

\section{Postsurgical phase}

Postoperative instructions including: Extra-oral ice packs during the first day and maintain daily routine oral hygiene after surgery and Patients were instructed to eat a soft diet for 7 days.

All patients received Postoperative medications including

- Broad-spectrum oral antibiotics: Amoxicillin $875 \mathrm{mg}$ / Clavulanic acid 125mg (Augmentin 1 gm Tablets, Medical Union Pharmaceuticals (MUP), GlaxoSmithKline, Cairo, Egypt) in a dose of one capsule twice daily for a week

- Non-steroidal anti-inflammatory drugs Ibuprofen $400 \mathrm{mg}$ (Brufen tablet 400mg Abbott, Cairo, Egypt) at a dose of one tablet three times daily for four days.

- Chlorhexidine $0.12 \%$ mouthrinses were prescribed for 2 weeks to enhance plaque control.

Sutures were removed one week postoperatively.

\section{Prosthetic phase}

After three months, the provisional acrylic crowns were removed and final porcelain fused to metal restoration were cemented over the abutments and functional loading was applied on the osseointegrated implants.

\section{Follow up phase}

- Clinical evaluation was performed for each patient immediately after implant placement and at set intervals of 1,3 and 6 months postoperatively. Patients were evaluated clinically for Presence of pain, tenderness or discomfort using the Numerical rating scale (NRS) (16), Presence of Swelling or infection and Implant Mobility according to the Clinical implant mobility scale developed by Mckinney and Koth (17). Clinical mobility was tested using back and forth pressure of approximately $500 \mathrm{gm}$ by two instrument handles.

- Radiographic evaluation:

Standardized periapical radiographs were obtained using a long cone paralleling technique, with the central beam perpendicular to the alveolar crest (XCP holder Rinn ${ }^{\mathrm{TM}}$, Dentsply International, York, PA, USA). Each X-ray holder was individualized with an occlusal record to standardize the procedure. Radiographs were taken immediately after implant placement, to serve as a baseline for measurement and at 1,3 and 6 months followup intervals (Fig 4, 5). All exposures were made with VARIO $^{\text {DG }}$ Dental X-Ray Machine (Sirona Dental Systems $\mathrm{GmbH}$, Bensheim, Germany) at $70 \mathrm{Kv}$ and 10 ma with 0.40-s exposure time and standardized periapical films (Ecta Speed; Kodak Co., NY, USA). The exposed films were developed in an automatic film processor with 6-min cycle at $27^{\circ} \mathrm{C}$. Indirect digital radiographic imaging using Standardized-scanning procedures was done for each radiograph. All radiographs were digitalized and stored in TIFF format. Measurements were performed with the aid of Image J software (Image J 1.31; A public image processing domain software, National Institute of Health, Bethesda, Maryland, USA) (18) used for assessment of bone density and marginal bone height around the implant and implant length was used for calibration.

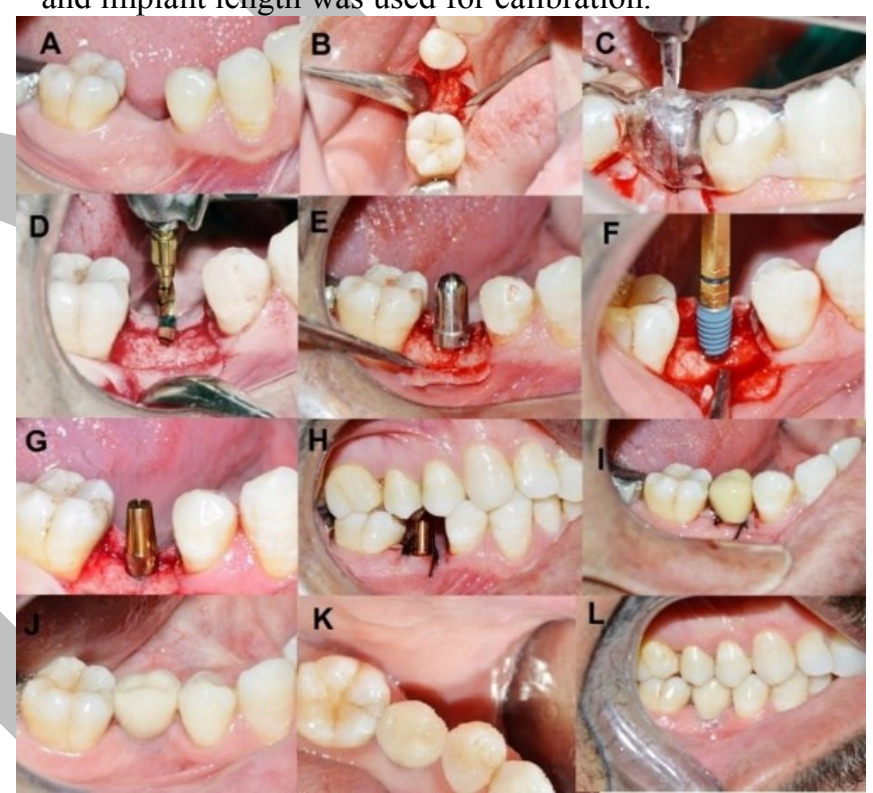

Figure 2: Representative clinical case (1): (A) Clinical Preoperative view of the ridge. (B) Incision and flap reflection. (C) Pilot drilling through acrylic surgical stent. (D) Preparation of the implant site. (E) Direction indicator in place. (F) Implant placement. (G) Abutment insertion. (H) Suturing. (I) Temporary restoration in position. (J) Final porcelain restoration three months postoperative. (K) Final porcelain restoration (occlusal view). (L) Final restoration after six months of loading

Assessment of the bone density around the implant: Image $\mathrm{J}$ software was used to evaluate radiographic bone density mesial and distal to each implant. The mean value of these two measurements was calculated for each implant.

Marginal bone height was determined on both mesial and distal implant surfaces using the linear measurement system supplied by the specially designed Image $\mathrm{J}$ software. Marginal bone level (MBL) was defined as the distance between a reference point (the implant shoulder) and the first marginal bone-to-implant contact level (19-21). Variations in bone level over time were defined as marginal bone change. Percent change in bone level was calculated based on the proportional difference between the implant length and the height from the crestal bone level to the implant apex. The mean value of these two measurements was calculated for each implant. 


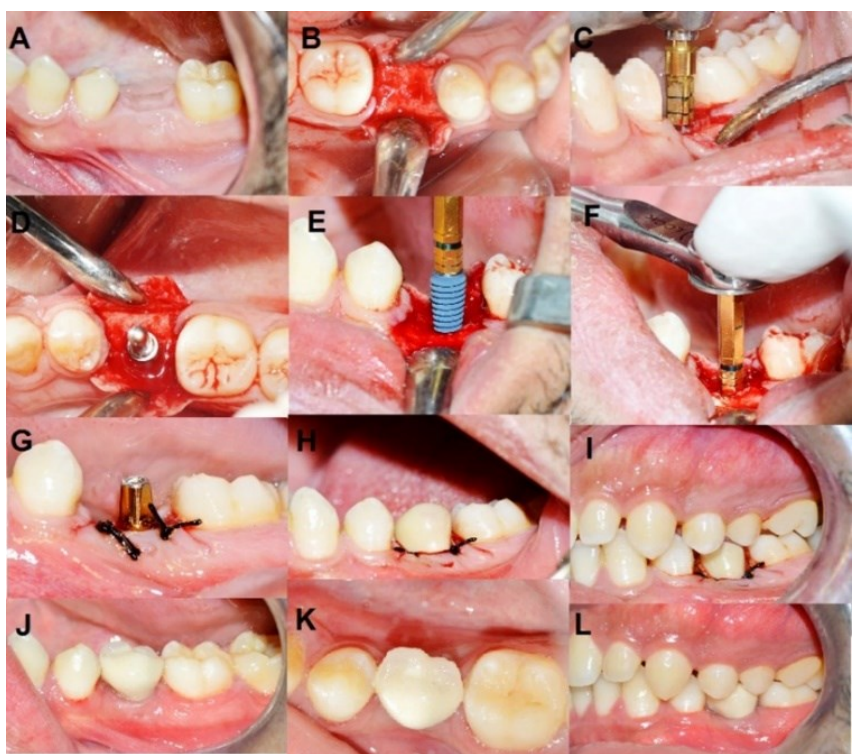

Figure 3: Representative clinical case (2) (A) Clinical Preoperative view of the ridge. (B) Incision and flap reflection.

(C) Drilling with subsequent drills. (D) Direction indicator (occlusal view). (E) Implant placement. (F) Complete insertion of the implant with Ratchet wrench. (G) Suturing. (H)Temporary restoration in position. (I) Temporary restoration free from occlusion. (J) Final porcelain restoration three months postoperative. (K) Final porcelain restoration (occlusal view). (L) Final restoration after six months of loading.

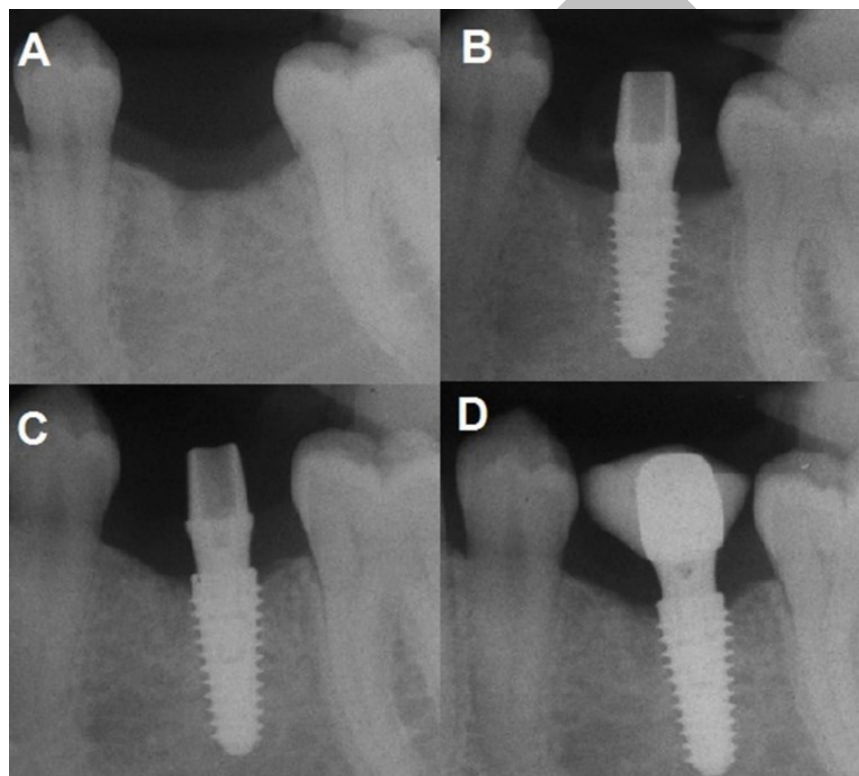

Figure 4: Radiographic follow up of case (1) a) Preoperative periapical radiograph of the implant site. b) Immediate postoperatively. c) Third month postoperativel d) Sixth month postoperatively.

\section{STATISTICAL ANALYSIS}

Statistical analysis was performed using IBM SPSS software package version 20.0 (22). Descriptive statistics for the study variables were performed including minimum, maximum, mean, and standard deviation. Repeatedmeasures ANOVA test were used for data analysis and comparison of differences between the mean values of periimplant bone density and radiographic marginal bone level changes from one interval time to another. Statistical significance was set at $p \leq 0.05$. Line charts were used for graphical representation of values in different interval times of follow-up.

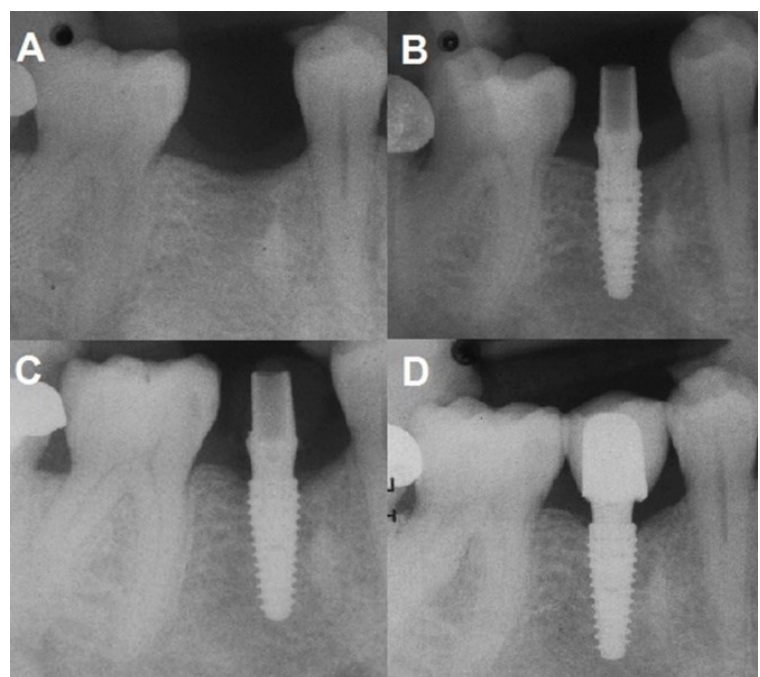

Figure 5: Radiographic follow up of case a) Preoperative periapical radiograph of the implant site. b) Immediate postoperatively c) Third month postoperatively d) Sixth month postoperatively.

\section{RESULTS}

Ten patients with extracted mandibular single-rooted teeth indicated for implant placement with immediate loading protocol were evaluated in this study. Their ages ranged from 20 to 40 years with mean age of 31.6 years. The ratio between males and females was 2: 3 (four males and six females). Three implants were inserted in the canine region having diameters of $3.5 \mathrm{~mm}$ and $4 \mathrm{~mm}$ and lengths of 11.5 $\mathrm{mm}$ and $13 \mathrm{~mm}$. Three implants were inserted in the first premolar region having diameters of 3.5, 4 and $4.5 \mathrm{~mm}$ and lengths of $10 \mathrm{~mm}$ and $11.5 \mathrm{~mm}$. Four implants were inserted in the second premolar region having diameters of 3.5, 4 and $4.5 \mathrm{~mm}$ and lengths of 10 and $11.5 \mathrm{~mm}$.

All patients were followed up for six months and the results were registered as regards: clinical evaluation and radiographic evaluation.

\section{I- Clinical evaluation}

1. Presence of pain, tenderness or discomfort: On the first postopertive day, all patients experienced mild to moderete pain at the surgical site scoring between three,four and five on NRS. Postopertive tenderness and discomfort were minimal in all cases. On the seventh postopertive day, all patients felt no pain, tenderness, discomfort except one case that felt moderate postoperative pain and discomfort in the first week after implant placement. After that all patients did not express pain or tenderness all over the follow up period. 2. Presence of Swelling or infection: In only one case periimplant infection with suppuration and swelling in the operated area was found after three weeks of implant placement. In the other cases, patients continued the follow up period without any clinical signs of inflammation, swelling or peri-implant infections after implant placement or during the evaluation period.

3. Implant Mobility: One implant showed grade III mobility registered in the first follow up month, that was regarded as a failure case and the implant was removed. None of the other implants showed any signs of mobility all over the evaluation period i.e. mobility score was 0 .

\section{II- Radiographic Evaluation:}

Mean peri-implant bone density values and standard deviation immediately post-operative, at $1^{\text {st }}$ month, at $3^{\text {rd }}$ month and at $6^{\text {th }}$ month were collected and tabulated (table 1, fig 6). 
Immediately post-operative, the mean peri-implant bone density value of all patients was $88.61 \pm 9.69$ with minimum recorded value of 67.20 and maximum recorded value of 98.27.

On the first month, the mean peri-implant bone density value of all patients was $108.93 \pm 7.14$ with minimum recorded value of 96.70 and maximum recorded value of 120.40. The increase in bone density value from immediately post-operative to first month was found to be statistically significant $\left(\mathrm{P}_{\text {Base }}<0.001\right)$.

On the third month, the mean peri-implant bone density value was $119.86 \pm 6.79$ with minimum recorded of 110.20 and maximum recorded value of 129.27 . The increase in bone density value from first month to third month was found to be statistically significant $\left(\mathrm{p}_{1}<0.001\right)$. Also, the increase in bone density value from immediately postoperative to the third month was found to be statistically significant $\left(\mathrm{P}_{\text {Base }}<0.001\right)$.

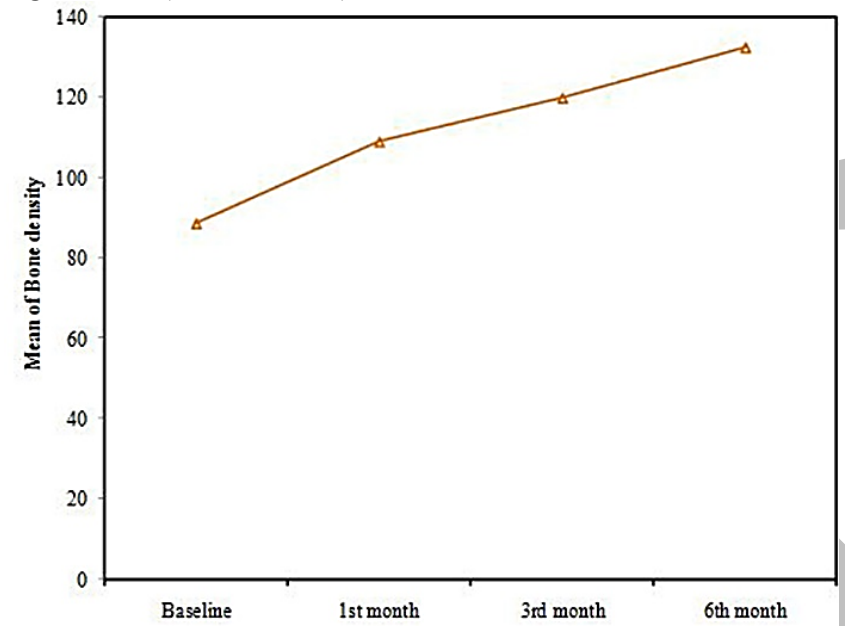

Figure 6: Assessment of bone density around the implant throughout the study period

On the sixth month, the mean peri-implant bone density value was $132.38 \pm 8.93$ with minimum recorded value of 117.94 and maximum recorded value of 143.35 . This increase in bone density value from third month to sixth month was found to be statistically significant $\left(\mathrm{P}_{3}=0.001\right)$.

The increase in bone density value from immediately postoperative to the $6^{\text {th }}$ month was found to be statistically significant $\left(\mathrm{P}_{\text {Base }}<0.001\right)$. Also, the increase in bone density value from first month to third month was found to be statistically significant $\left(\mathrm{p}_{2}<0.001\right)$.

The mean marginal bone level values and standard deviation immediately post-operatively, at $1^{\text {st }}$ month, at $3^{\text {rd }}$ month and at $6^{\text {th }}$ month are shown in (Tables 2).

Immediately postoperative, the mean MBL value was $0.16 \pm 0.06$ with a minimum recorded value of 0.07 and a maximum recorded value of 0.26 .

On the first month, the mean MBL value was $0.46 \pm 0.18$ with minimum-recorded value of 0.15 and maximum-recorded value of 0.68 . This difference in MBL values from immediately postoperative to first month was found to be statistically significant

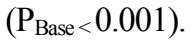

On the third month, the mean MBL value was $0.65 \pm 0.25$ with minimum recorded value of 0.20 and maximum recorded value of 0.90 . The difference in MBL values from immediately post-operative to the third month was found to be statistically significant $\left(\mathrm{P}_{\mathrm{Base}}<0.001\right)$ and The difference from the first month to the third month was found to be statistically significant $(\mathrm{p} 1=0.037)$

On the sixth month, the mean MBL value was $0.58 \pm 0.1$ with minimum recorded value of 0.25 and maximum recorded value of 0.79 . The difference in MBL values from immediately post-operative to the sixth month was found to be statistically significant $\left(\mathrm{P}_{\text {Base }}<0.001\right)$ The difference from first month to sixth month was found to be statistically significant $\left(\mathrm{p}_{2}=0.008\right)$. The difference in MBL value from third month to sixth month was found to be statistically insignificant $\left(\mathrm{p}_{3}=0.254\right)$.

Table (1): Descriptive statistics of bone density values around the implants throughout the study period.

\begin{tabular}{|c|c|c|c|c|}
\hline & $\begin{array}{c}\text { Immediate } \\
\text { postoperative } \\
(n=10)\end{array}$ & $\begin{array}{c}1^{\text {st }} \\
\text { month } \\
(n=9)\end{array}$ & $\begin{array}{c}3^{\text {rd }} \\
\text { month } \\
(n=9)\end{array}$ & $\begin{array}{c}6^{\text {th }} \\
\text { month } \\
(n=9)\end{array}$ \\
\hline \multicolumn{5}{|l|}{$\begin{array}{c}\text { Bone } \\
\text { density }\end{array}$} \\
\hline Min & 67.20 & 96.70 & 110.20 & 117.94 \\
\hline Max. & 98.27 & 120.40 & 129.27 & 143.35 \\
\hline $\begin{array}{l}\text { Mean } \\
\pm \text { SD. }\end{array}$ & $88.61 \pm 9.69$ & $\begin{array}{l}108.93 \\
\pm 7.14\end{array}$ & $\begin{array}{l}119.86 \\
\pm 6.79\end{array}$ & $\begin{array}{l}132.38 \\
\pm 8.93\end{array}$ \\
\hline Median & 90.68 & 109.45 & 119.86 & 134.70 \\
\hline $\mathbf{p}_{\text {Base }}$ & & $<0.001^{*}$ & $<0.001^{*}$ & $<0.001^{*}$ \\
\hline $\begin{array}{l}\text { Sig. bet. } \\
\text { periods }\end{array}$ & \multicolumn{4}{|c|}{$\mathrm{p}_{1}<0.001^{*}, \mathrm{p}_{2}<0.001^{*}, \mathrm{p}_{3}=0.001^{*}$} \\
\hline
\end{tabular}

$\mathrm{P}_{\mathrm{Base}} \mathrm{p}$ value for $\mathrm{F}$ test (ANOVA) with repeated measures (LSD) for comparing between Baseline with each other period

$\mathrm{p}_{1}: \mathrm{p}$ value for $\mathrm{F}$ test (ANOVA) with repeated measures (LSD) for comparing between $1^{\text {st }}$ month and

$3^{\text {rd }}$ month

$\mathrm{p}_{2}: \mathrm{p}$ value for $\mathrm{F}$ test (ANOVA) with repeated measures (LSD) for comparing between $1^{\text {st }}$ month and $6^{\text {th }}$ month

$\mathrm{p}_{3}: \mathrm{p}$ value for $\mathrm{F}$ test (ANOVA) with repeated measures (LSD) for comparing between $3^{\text {rd }}$ month and $6^{\text {th }}$ month

*: Statistically significant at $\mathrm{p} \leq 0.05$

\section{DISCUSSION}

The implant design plays an even more important role if surgical protocols providing immediate loading are adopted (23). A newly developed implant with a specific macrodesign of knife threads geometry has been developed that is claimed to simplify immediate loading protocols. Therefore, the present study was designed to clinically and radiographically evaluate the immediately loaded knife thread implants for the replacement of mandibular singlerooted teeth.

In this study, 10 patients with missing mandibular single rooted teeth were selected from the Outpatient Clinic of the Oral and Maxillofacial Surgery Department, Faculty of Dentistry, Alexandria University. The selected patients were free from parafunctional habits such as bruxism or clenching to avoid the high magnitude of horizontal forces (rather than axial) that can put undesirable load on the implants (24). These criteria were in agreement with the clinical recommendations of Schwarz et al in 2016 (25) that immediate loading protocols should be avoided in patients with bruxism and clenching. 
In the present study, tapered implant design with narrow apical diameter and knife thread design was used. This design increased cutting efficiency during insertion as it cut through the bone smoothly and condense it simultaneously and offered higher primary stability (13).

Table (2): Descriptive statistics marginal bone height values around the implants throughout the study period.

\begin{tabular}{|c|c|c|c|c||}
\hline & $\begin{array}{c}\text { Baseline } \\
(\mathbf{n = 1 0 )}\end{array}$ & $\begin{array}{c}\mathbf{1}^{\text {st }} \\
(\mathbf{n}=\mathbf{9})\end{array}$ & $\begin{array}{c}\mathbf{3}^{\text {rd }} \\
\text { month } \\
(\mathbf{n}=\mathbf{9})\end{array}$ & $\begin{array}{c}\mathbf{6}^{\text {th }} \\
\text { month } \\
(\mathbf{n}=\mathbf{9})\end{array}$ \\
\hline & & & & \\
Min. - & $0.07-$ & $0.15-$ & $0.20-$ & $0.25-$ \\
Max. & 0.26 & 0.68 & 0.90 & 0.79 \\
$\begin{array}{c}\text { Mean } \\
\pm \text { SD. }\end{array}$ & $0.16 \pm$ & $0.46 \pm$ & $0.65 \pm$ & $0.58 \pm$ \\
Median & 0.16 & 0.18 & 0.25 & 0.17 \\
& 0.48 & 0.73 & 0.61 \\
\hline pase & & $<0.001^{*}$ & $<0.001^{*}$ & $<0.001^{*}$ \\
\hline $\begin{array}{c}\text { Sig. bet. } \\
\text { periods }\end{array}$ & \multicolumn{2}{|c|}{$\mathrm{p}_{1}=0.037^{*}, \mathrm{p}_{2}=0.008^{*}, \mathrm{p}_{3}=0.254$} \\
\hline
\end{tabular}

$\mathrm{P}_{\text {Base }}$ : $\mathrm{p}$ value for $\mathrm{F}$ test (ANOVA) with repeated measures (LSD) for comparing between Baseline with each other period

$\mathrm{p}_{1}$ : $\mathrm{p}$ value for $\mathrm{F}$ test (ANOVA) with repeated measures (LSD) for comparing between $1^{\text {st }}$ month and

$3^{\text {rd }}$ month

$\mathrm{p}_{2}$ : $\mathrm{p}$ value for $\mathrm{F}$ test (ANOVA) with repeated measures (LSD) for comparing between $1^{\text {st }}$ month and

$6^{\text {th }}$ month

$\mathrm{p}_{3}: \mathrm{p}$ value for $\mathrm{F}$ test (ANOVA) with repeated measures (LSD) for comparing between $3^{\text {rd }}$ month and

$6^{\text {th }}$ month

*: Statistically significant at $\mathrm{p} \leq 0.05$

Regarding the implant surface topography, a nanostructured calcium-incorporated surface (XPEED surface) was used in the present study. As it has a nanolayer of calcium ions incorporated on the sandblasted, large-grit, acid-etched (SLA) surface. The ions create a $\mathrm{CaTiO} 3$ nanostructure on the surface and activate osteoblasts in the living bone for fast and strong osseointegration. The Titanium implants with XPEED surfaces significantly enhanced apatite formation, the $\mathrm{BIC} \%$ and may induce strong bone integration by improving osseointegration of grit-blasted Titanium implants in areas of poor quality bone (15).

As regards the surgical procedure, all included patients were subjected to delicate and atraumatic surgery using immediate loading protocol. All implants were inserted according to the manufacturer's recommendations, at crestal level. Primary stability was recorded in terms of insertion torque, which was adjusted at least $35 \mathrm{Ncm}$ in agreement with Clinical recommendations regarding the immediate implant loading protocols of Schwarz et al (25). The control of an adequate insertion torque has been recommended by Kim et al in 2015 (26) as they suggested that an insertion torque greater than $32 \mathrm{Ncm}$ was essential to accomplish osseointegration and considered as an appropriated approach to minimize implant failure related to the inadequate primary stability of immediately loaded implants.

In the present study, immediate non-occlusal loading protocol was selected. All implants were immediately restored with provisional crowns without direct occlusal contacts with the opposing dentition, both in static or dynamic lateral movements. That non-occlusal pattern of loading results in lesser risk of implant failure than the occlusal pattern in accordance to Sanz-Sánchez et al. findings in 2015 (27). That the presence of occlusal contacts may lead to micromotion at the bone-implant interface if not adequately controlled and ultimately cause fibrous encapsulation and implant failure.

From our clinical evaluation throughout the follow up period, which was extended up to 6 months, only one implant was lost, in the canine region of a female patient, within the healing period before the delivery of the definitive metal-ceramic restoration. The failed implant displayed infection with pus and swelling in the operated area three weeks after implant placement. It showed grade III mobility one month after surgery and needed removal. As reported by Al-Sabbagh et al in 2015 (28) that the clinical signs of peri-implant infection are considered to be associated with implant failures. The lack of osseointegration is generally distinguished by implant mobility and radiological radiolucency. Here, the implant is considered to be failed. Failure of this case could be attributed to lack of oral hygiene maintenance by the patient in spite of the instructions given to her. Also early infection around the implant may have a role in preventing osseointegration.

All the other implants were stable with no clinical mobility in any of them throughout the follow up period. This was confirmed by the radiographic evaluation that revealed intimate bone- implant contact and absence of periimplant radiolucency. Also, none of the other implants showed any persistent pain, discomfort, tenderness, infection or swelling.

The present study showed statistically significant increase in peri-implant bone density from the immediate postoperative period to the end of the 6 months of the evaluation period, which indicates successful osseointegration around the implants.

These findings agreed with the conclusions of Ramachandran et al in 2015 (29) who reported that immediate loading of implants helped stimulate bone ossification around implants at the lateral apical level at 3 and 6 months and at the crest from 3 months to 6 months. Furthermore, the immediate nonfunctional loading of implants resulted in a significantly lesser degree of bone demineralization at the alveolar crest from the time of implant placement up to 3 months compared with immediate functional loading. These results also may be attributed to nanostructured calcium-incorporated surface of the implants used in the present study which is osteoconductive and may promote bone healing, these findings agreed with the results of Felice et al (14).

Regarding peri-implant bone level, there was statistically significant difference in the mean of peri-implant bone level changes from immediate postopertive till the $3^{\text {rd }}$ month. This increase was statistically significant. The difference from the 3rd month to 6th month was statistically insignificant but the change of MBL from immediate postoperative to 6 months was statistically significant.

A recent study by Luongo et al in 2014 (13) showed that patients had lost a mean of $0.32 \mathrm{~mm}( \pm 0.22)$ of peri-implant marginal bone after 1 year of functional loading of the same knife thread implant design The authors stated also" the use of a fixture with specific macroscopical features may be helpful to maximize initial stability in immediate loading cases". 
In another case report of Mangano et al in 2015 (30) using the same implant design stated that "The threads of the implants used in the study were designed to provide high insertion torque, by increasing their dimensions toward the coronal end of the implant. This specific macrotopographical feature may allow for axial and radial bone compression during implant insertion, and it may be particularly useful in regenerated areas, providing the increased primary stability. Also, An insertion torque of $55 \mathrm{Ncm}$ was registered with that knife thread implant design.

\section{CONCLUSIONS}

Within the limits of this study, the overall conclusion that can be drawn from this study is that immediate non-occlusal loading of a knife thread implant in the mandibular single rooted teeth is a predictable treatment procedure, with good survival rates $(90 \%)$ and a low incidence of complications. In other words, the specially designed knife thread implant design may have minimized the risk of failure of immediately loaded single implants

As immediate loading reduces the treatment time and could offer more comfort for the patient, this strategy should be considered as an alternative to conventional loading. However, the concept of immediate loading should be performed according to a specified protocol with attention to adequate primary implant stability and careful patient instruction.

\section{CONFLICT OF INTERSET}

The authors declare that they have no conflicts of interest.

\section{REFERENCES}

1. Chaushu G, Chaushu S, Tzohar A, Dayan D. Immediate loading of single-tooth implants: immediate versus nonimmediate implantation. Int J Oral Maxillofac Implants 2001; 16: 267-72.

2. CheeW, Jivraj S. Efficiency of immediately loaded mandibular full-arch implant restorations. Clin Oral Impl Res 2003; 5: 52-6.

3. Esposito M, Grusovin MG, Achille H, Coulthard P, Worthington HV. Interventions for replacing missing teeth: different times for loading dental implants. Cochrane Database Syst Rev 2009; 1:1-38.

4. Hui E, Chow J, Li D, Liu J, Wat P, Law H. Immediate provisional for single-tooth implant replacement with Brånemark system: preliminary report. Clin Implant Dent Relat Res 2001; 3: 79-86.

5. Nelson K, Semper W, Hildebrand D, Özyuvaci H. A retrospective analysis of sandblasted, acid-etched implants with reduced healing times with an observation period of up to 5 years. Int J Oral Maxillofac Implants 2008; 23: 72632 .

6. O'Sullivan D, Sennerby L, Jagger D, Meredith N. Comparison of two methods of enhancing implant primary stability. Clin Implant Dent Relat Res 2004; 6: 48-57.

7. Szmukler-Moncler S, Salama H, Reingewirtz Y, Dubruille JH. Timing of loading and effect of micro-motion on boneimplant interface. A review of experimental literature. J Biomed Mat Res 1998; 43: 192-203.

8. Bilhan H, Geckili O, Mumcu E, Bozdag E, Sünbüloðlu E, Kutay O. Influence of surgical technique, implant shape and diameter on the primary stability in cancellous bone. J Oral Rehabil 2010; 37: 900-7.
9. Meltzer AM. Primary stability and initial bone-to-implant contact: The effects of immediate placement and restoration of dental implants. J Implant Reconstr Dent 2009; 1: 35-41.

10. Torroella-Saura G, Mareque-Bueno J, Cabratosa-Termes J, Hernández-Alfaro F, Ferrés-Padró E, Calvo-Guirado JL. Effect of implant design in immediate loading. A randomized, controlled, split-mouth, prospective clinical trial. Clin Oral Impl Res 2015; 26: 240-4.

11. Misch, C.E., Wang, H.L., Misch, C.M., Sharawy, M., Lemons, J. \& Judy, K.W. Rationale for the application of immediate load in implant dentistry: part II. Implant Dentistry 2004; 13: 310-21.

12. Orsini E, Salgarello S, Bubalo M, Lazic Z, Trire A, Martini D, et al. Histomorphometric evaluation of implant design as a key factor in peri-implant bone response: a preliminary study in a dog model. Minerva Stomatologica 2009; 58: 263-75.

13. Luongo G, Lenzi C, Raes F, Eccellente T, Ortolani M, Mangano C. Immediate functional loading of single implants: a 1-year interim report of a 5-year prospective multicentre study. Eur J Oral Implantol 2014; 7: 1-13.

14. Felice P, Grusovin MG, Barausse C, Grandi G, Esposito M. Safety and effectiveness of early loaded maxillary titanium implants with a novel nanostructured calcium-incorporated surface (Xpeed): 3-year results from a pilot multicenter randomised controlled trial. Eur J Oral Implantol. 2015; 8: 245-54.

15. Lee SY, Yang DJ, Yeo S, An HW, Ryoo KH, Park KB. The cytocompatibility and osseointegration of the Ti implants with XPEED $^{\circledR}$ surfaces. Clin Oral Impl Res 2012; 23: 1283-9.

16. Farrara JT, Young JP Jr., LaMoreauxb L, Werthb JL, Poole RM. Clinical importance of changes in chronic pain intensity measured on an 11-point numerical pain rating scale. Pain 2001; 94: 149-58.

17. McKinney R, Koth DL, Steflik D, Robinson F, Davis B, Morris $\mathrm{C}$, et al. Prospective investigation of single-crystal sapphire endosteal dental implant in humans: Ten year results. J Oral Implantol 1995; 21: 8-18.

18. Abramoff MD, Magelhaes PJ, Ram SJ. Image Processing with Image J. Biophotonics Int 2004; 11: 36-42.

19. Dias DR, Leles CR, Lindh C, Ribeiro-Rotta RF. The effect of marginal bone level changes on the stability of dental implants in a short-term evaluation. Clin. Oral Impl. Res. 2015; 26:1185-90.

20. Burtscher D, Norer B, Dalla Torre D, Beier U, Schubert K, Grunert I. A 7-year Prospective Radiographic Evaluation of marginal bone level around two different implant systems: a randomized clinical trial. Clin Oral Impl Res 2015; 26 : 1244-9.

21. Berberi AN, Sabbagh JM, Aboushelib MN, Noujeim ZF, Salameh ZA. A 5-year comparison of marginal bone level following immediate loading of single-tooth implants placed in healed alveolar ridges and extraction sockets in the maxilla. Front Physiol 2014; 5:1-7.

22. Kirkpatrick LA, Feeney BC. A simple guide to IBM SPSS statistics for version 20.0. Student ed. Belmont, Calif.: Wadsworth, Cengage Learning, 2013.

23. Esposito M, Coulthard P, Thomsen P, Worthington HV. The role of implant surface modifications, shape and material on the success of osseointegrated dental implants. A Cochrane systematic review. Eur J Prosthodont Restor Dent 2005; 13: 15-31.

24. Porter JA, von Fraunhofer JA. Success or failure of dental implants? A literature review with treatment considerations. Gen Dent 2005; 53: 423-32. 
25. Schwarz F, Sanz-Martın I, Kern J-S, Taylor T, Schaer A, Wolfart S, et al. Loading protocols and implant supported restorations proposed for the rehabilitation of partially and fully edentulous jaws. Camlog Foundation Consensus Report. Clin Oral Impl Res 2016.

26. Kim TH, Knezevic A, Jorgensen M, Rich S, Nowzari H. Prospective, 1-year observational study of doublethreaded tapered body dental implants with immediate loading. J Prosthet Dent 2015; 114: 46-51.

27. Sanz-Sánchez I, Sanz-Martín I, Figuero E, Sanz M. Clinical efficacy of immediate implant loading protocols compared to conventional loading depending on the type of the restoration: a systematic review. Clin Oral Implants Res 2015; 26: 964-82.

28. Al-Sabbagh M, Bhavsar I. Key local and surgical factors related to implant failure. Dent Clin North Am 2015; 59: 123.

29. Ramachandran A, Singh K, Rao J, Mishra N, Jurel SK, Agrawal KK. Changes in alveolar bone density around immediate functionally and nonfunctionally loaded implants. J Prosthet Dent 2015; 11: 1-6.

30. Mangano FG, Zecca PA, van Noort R, Apresyan S, Iezzi G, Piattelli A, et al. Custom-Made Computer-AidedDesign/Computer-

Aided-Manufacturing Biphasic Calcium-Phosphate Scaffold for Augmentation of an Atrophic Mandibular Anterior Ridge. Case Rep Dent 2015; 2015: 941265. 